\title{
ESPbase: A Microsoft Access tool for selecting symbol and icon sets for usability
}

\author{
OSCAR DE BRUIJN and SINÉ MCDOUGALL \\ University of Wales Swansea, Swansea, Wales \\ and \\ MARTIN B. CURRY \\ Sowerby Research Centre, British Aerospace, Bristol, England
}

\begin{abstract}
The ESPbase provides a tool for storing symbols and icons along with information about their characteristics. Information about a wide range of symbol characteristics is included on the database to facilitate the selection of symbol sets for research and design. The database includes information about the graphical characteristics and functions of symbols. It also includes ratings of symbol concreteness, complexity, familiarity, and meaningfulness. Symbols and icons can be accessed on the basis of each of these characteristics or any combination of characteristics. This makes it easier to select symbols on the basis of usability and design requirements. It also means that symbols can be easily selected for research while controlling their characteristics on a number of dimensions.
\end{abstract}

A range of normative data are available for words (see, e.g., Benjafield, Frommhold, Keenan, Muckenheim, \& Mueller, 1993; Friendly, Franklin, Hoffman, \& Rubin, 1982; Gilhooly \& Logie, 1980; Paivio, Yuille, \& Madigan, 1968) and pictures (Martein, 1995; Sanfeliu \& Fernandez, 1996; Snodgrass \& Vanderwart, 1980; van Schagen, Tamsma, Bruggemann, Jackson, \& Michon, 1983) in order to control their characteristics when such items are used as experimental stimuli in psycholinguistic research. Norms available for words include their concreteness, familiarity, and meaningfulness, while those available for pictures include their familiarity, meaningfulness, and name agreement. These characteristics of word and picture stimuli often need to be controlled in order to avoid confounding them with the characteristic(s) under investigation. Recently, normative ratings were collected for the concreteness, complexity, familiarity, meaningfulness, semantic distance, name agreement, and concept agreement of a large number of symbols ${ }^{1}$ (McDougall, Curry, \& de Bruijn, 1999). These norms have been included in the database and can be used to control the characteristics of symbols used in symbol usability research. In addition, ratings of the semantic, syntactic, and pragmatic usability of a large number of symbols (American Institute of Graphic Arts [AIGA], 1982) have been included in the database and can be used when one is considering the usability and design of symbol sets.

This research was part of the Effective Symbology Project, which was supported by a grant from British Aerospace plc (Grant SRC/UOS/ 060495). Correspondence should be addressed to S. McDougall, Psychology Department, University of Wales Swansea, Swansea SA2 8PP, Wales (e-mail: s.mcdougall@, swansea.ac.uk).
There is now a growing body of research examining what characteristics of symbols affect their usability, and it appears that control over these characteristics may be as important to symbol usability research as control over word and picture characteristics is to psycholinguistic research. For example, it is often assumed that symbols, and particularly computer icons, are more easily processed when they are concrete than when they are abstract. Use of concrete symbols is supposed to be easier because they depict objects, places, and people with which we are already familiar in the real world, making their meanings more obvious than those of abstract symbols. However, despite the large number of studies that have investigated the effects of concrete versus abstract symbols on usability (Arend, Muthig, \& Wandmacher, 1987; Green \& Barnard, 1990; Rogers, 1986; Rogers \& Oborne, 1987; Rohr \& Keppel, 1985; Stammers, George, \& Carey, 1989; Stammers \& Hoffman, 1991), the precise circumstances under which concrete symbols are more usable than abstract symbols remains unclear (but see McDougall, Curry $\&$ de Bruijn, 1998). Part of the inconsistency concerning the effects of concreteness on usability found in these studies may be due to a lack of control over the characteristics of the symbols used in these studies other than their concreteness. For example, in some of this research, the concreteness of symbols may have been confounded with their complexity (Garcia, Badre, \& Stasko, 1994). In order to examine the effects of concreteness on usability, it is important to be able to select symbols on the basis of their concreteness while controlling for their complexity. Indeed, it may be generally important to control the characteristics of symbols used in research in order to avoid confounding their effects on usability.

Controlling symbol characteristics is also important whenever there is a need to select symbols to be used in 
new applications (e.g., Barnard \& Marcel, 1984; Dyson, 1992; Gittens, 1986). As symbol usability research provides links between an ever-growing number of symbol characteristics and their usability, it may be possible to predict the usability of symbols relative to a number of alternatives on the basis of their characteristics. Thus, whenever there is reason to believe that symbols with certain characteristics can increase the usability of an application relative to other symbols, it may be desirable to select symbols possessing these characteristics. For example, it has been argued that usability of symbols with similar meanings is enhanced by the use of common graphical elements such as color (e.g., Christ, 1984) and shape (e.g., Gittens, 1986). On the other hand, there is some evidence to suggest that symbol usability is enhanced by the use of unique graphical elements that make symbols more discriminable (e.g., Blackwell \& Cuomo, 1991; Geiselman, Landee, \& Christen, 1982). If the circumstances under which usability is enhanced by common or unique graphical elements are known, this knowledge could be used to select symbol sets on the basis of their graphical characteristics to best fit the circumstances in which they will be used.

Given the large number of characteristics that may need to be considered, the task of selecting symbols for symbol usability research or for designing new applications would be greatly facilitated by a computerized database in which symbols are stored together with information about a large number of their characteristics. Symbol sets can then be accessed by simply specifying their desired characteristics. However, despite the availability of a large number of collections of symbols accessible by computer, particularly on the World-Wide Web (e.g., CrystalVision, 1994; Graphic Artists Guild Foundation, 1995; Gray, 1990; Horton, 1994; The Icon Factory, 1997), none of these provide facilities for the retrieval of symbols on the basis of their characteristics. Even if some structure is apparent in the way symbols are stored in these collections, this structure is usually based on just a single symbol characteristic, such as, for example, symbol size (e.g., $16 \times 6$ pixels), file type (e.g., tiff, jpeg, or bitmap), function (e.g., "delete," "save"), or application (e.g., Microsoft Windows 95, Apple Macintosh). A collection in which symbols are stored according to a single symbol characteristic allows symbols to be retrieved in only one way, whereas for symbol usability research and design purposes, it is important that symbols can be retrieved depending on many different characteristics. Thus, any computerized database that allows symbols to be accessed on the basis of many different characteristics will inevitably be more useful for research and design purposes than one that allows symbols to be selected on the basis of only a single characteristic.

Computerized retrieval of words on the basis of a large number of their characteristics has been available for psycholinguistic research for several years (see Coltheart, 1981; Quinlan, 1992; Wilson, 1988). The Oxford Psycho- linguistic Database (Quinlan, 1992) provides a computerized way of selecting word sets, taking into account the syntactic, semantic, phonological, and orthographic information of the words. Available in this database are properties of words such as their number of syllables, their regularity or irregularity of spelling, and their frequency of occurrence in printed English. The Oxford Psycholinguistic Database also includes the norms for the concreteness, familiarity, and meaningfulness, which are available for a large number of words. Each of these characteristics can be used to select words in the database simply by specifying criteria to which these words need to comply. For example, the inclusion of information about word type, frequency of occurrence, and concreteness of words stored in the database allows all concrete nouns with a frequency of occurrence of 500 per million or more to be selected from the list of words for which this information is specified.

The need to select symbols with certain characteristics for both research and design purposes, together with the recent development of norms for a large number of symbols, prompted the development of the Effective Symbology Project Symbol Database (ESPbase), which offers flexibility in the construction of symbol sets similar to that offered by the Oxford Psycholinguistic Database for the construction of word sets. However, unlike the Oxford Psycholinguistic Database, which allows only data retrieval, the ESPbase provides facilities for storing new symbols together with a large number of their characteristics. Information about each of these characteristics can be used to access these symbols at a later date. Thus, in addition to providing easy access to symbols already stored on the database, the ESPbase provides a way of structuring new symbol data in such a way that this information can be used in the construction of symbol sets for research and design purposes.

The ESPbase is implemented as a Microsoft Access application running in a Microsoft Windows environment. ${ }^{2}$ This provides several advantages in terms of ease of interacting with the database and flexibility in customizing the database to suit the users' needs. Microsoft Windows provides an easy way of interacting with the database for anyone with a basic knowledge of the Microsoft Windows graphical user interface. It utilizes different windows to display symbol information, to specify search criteria, and to display the results of symbol searches. Navigation between these windows occurs using menu bars, toolbars, and buttons that are inherent to the Microsoft Windows graphical interface. Using Microsoft Access as a platform for the ESPbase also has the advantage that all its functionality can be easily customized by anyone with a knowledge of building Microsoft Access applications.

In the next section, a description of the different symbol characteristics incorporated in the database is given together with the reasons why these particular characteristics were included. This is followed by a description of 
the different facilities incorporated in the database that allow sets of symbols conforming to a number of criteria to be selected.

\section{NATURE OF THE INFORMATION IN THE ESPbase}

Information in the ESPbase is displayed in the Data Form, shown in Figure 1. Because symbols are different from words, characteristics that allow effective symbol search are not necessarily the same as the word characteristics included in the Oxford Psycholinguistic Database. Information in the Data Form is divided into three sections: (1) symbol information (left section in Figure 1), (2) function information (upper-right section), and (3) ratings information (lower-right).

Precise details regarding the information contained in each section are given below.

\section{Symbol Information}

The Symbol Information section of the Data Form contains a graphical image of the symbol along with all the information directly concerning this image and its visual characteristics.

Symbol ID number. Each symbol is indexed by a Symbol ID number, which provides a unique reference to each of the symbols. All other information in the database is linked to the Symbol ID numbers. Thus, the Symbol ID number works as a key that links together all the separate pieces of information concerning one particular symbol.

Source. Symbols are drawn from a variety of different sources. These include standards organizations (e.g., the International Standards Organisation [ISO], the International Electrotechnical Committee [IEC], and the British Standards Institute $[\mathrm{BSI}])$, the AIGA, computer software packages (e.g., Microsoft Access, Microsoft Word, PaintShop Pro, etc.), Horton (1994), Dreyfuss (1971), and a variety of websites (e.g., Graphic Artists Guild Foundation, 1995; The Icon Factory, 1997). Source information allows symbols to be selected from particular sources. This may be useful when symbols are needed for use in applications for which symbols have already been developed or if the symbols have to be part of (international) standards.

Symbol image. Symbols are central to the database and are stored as graphical images. Symbols are saved as bitmap image files that are then embedded in the symbol database, from which they can be subsequently retrieved. ${ }^{3}$ This method of storing symbol images in the database ensures that images can be edited (using graphics software such as Microsoft Paintbrush or PaintShop Pro), copied to other applications (using Windows Clipboard function), or saved in separate files using different file formats.

Image contents. The symbol database contains a verbal description of each of the symbols' images present in the database. This field contains descriptions of the symbols in terms of what is depicted in the symbol rather than their graphical characteristics, which are described below. With this information, symbols can be retrieved from the database by specifying one or more keywords that describe the semantic contents of the symbol. For example, a symbol that is described as "a silhouette of a man viewed from the front" can be retrieved by using the keyword man.

Image characteristics. Given a large collection of graphic images, it is often desirable to retrieve these images on the basis of their graphical characteristics. This could be achieved by using, for example, mathematical image mapping routines (e.g., Liu \& Sun, 1997; Mehtre, Kankanhalli, \& Lee, 1997) or neural networks (e.g., Mazza, 1997), but we opted for providing a list of graphical features (Figure 2) that can be used to characterize symbols graphically. Dyson (1992) showed that most symbols can be categorized in accordance with a list of graphical features that the symbols either do or do not have. This means that it is possible to characterize a symbol by simply checking its features off on a list and using this characterization to retrieve symbols from the database. Thus symbols can be selected on the basis of individual features or sets of features. Furthermore, it is possible to avoid creating symbol sets that share too many similar features and therefore could become confusable.

A checklist with graphical features is available for each symbol in the Image Characteristics Box (Figure 2), which pops up when its button is clicked in the Data Form. Graphical features are divided into four types: (1) colors, which are separated into foreground and background colors; (2) global features, which are those characteristics concerning the entire image, such as, for example, symmetry and line drawing; (3) local features, which are the graphical elements from which an image is constructed, such as, for example, lines, circles, and boxes; (4) special features, each of which can be considered as carrying a certain meaning that is derived from the conventions that exist for their use, such as, for example, arrows, which are often used to indicate direction of movement.

\section{Function Information}

Unlike words, symbols are used to represent functions that are not necessarily directly related to whatever is depicted in the symbol. Indeed, in the evaluation of the factors underlying symbol usability, a distinction is often made between a symbol's primary referent (what is depicted in the symbol), its secondary referent (its function), and the closeness of the relationship between the symbol and its function (e.g., Familant \& Detweiler, 1993). Thus, a characterization of symbols is not complete without reference to their functions.

Information related to functions includes a unique reference number for each function (the Function ID number), the name of each function, and a longer description of the function if required (Figure 1). This information allows symbols to be selected on the basis of the function or type of function they have been used to represent. Functions can be represented by more than one symbol 


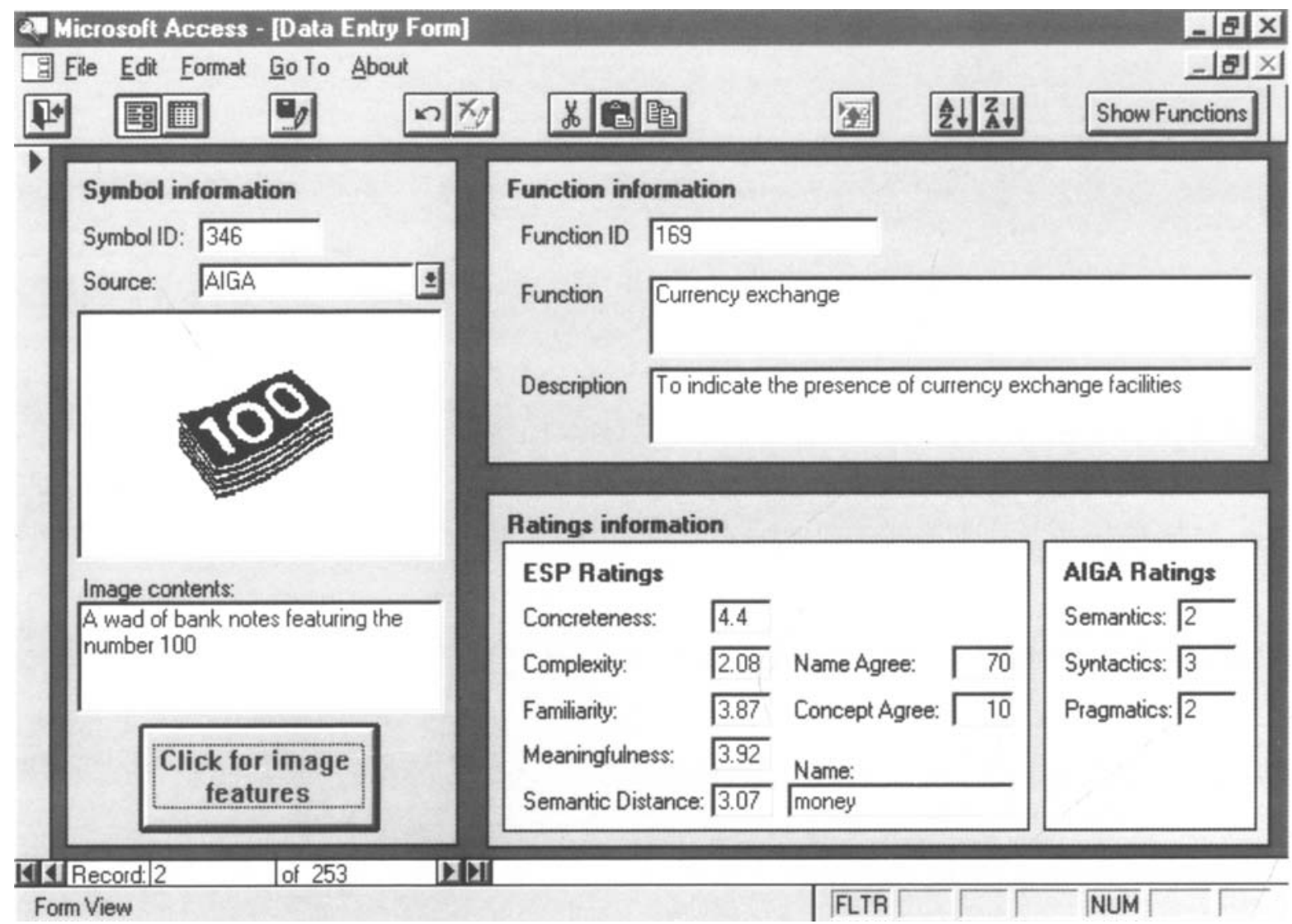

Figure 1. The ESPbase Data Form used to enter and view symbol, function, and ratings information.

so that when a function is used to select symbols in the database, all the symbols used to represent that particular function will be retrieved.

Function ID. Each function in the database has a Function ID number. All information concerning a particular function is linked together by means of its Function ID number.

Function names. Functions are identified by a function name. A function name consists of a verbal label (e.g., "save document" or "save" for writing documents to disk). These function names are the most common way of referring to functions.

Function descriptions. In addition to a function name, some functions are accompanied by a somewhat fuller description of the function. For example, the function labeled "tape recorder" is described in more detail as, "On amplifying and measuring equipment, to identify the terminals, switches, and controls by means of which a tape recorder is to be connected and operated."

\section{Ratings information}

The database also includes information about a number of visual and semantic dimensions of symbols (Figure 1) in the form of subjective ratings. Ratings on the database come from the Effective Symbology Project (ESP ratings), and the American Institute of Graphic Arts (AIGA ratings). Details of each of these ratings are given below.

ESP ratings. ESP ratings are available for a set of 239 symbols included in the database (see McDougall et al., 1998, for further details). Ratings have been obtained for symbol concreteness, familiarity, meaningfulness, visual complexity, and semantic distance. The ratings provide a measure of the degree to which a certain quality is present or absent in each symbol. All of the ratings were obtained using a $1-5$ scale $(1=$ largely absent, $5=$ strongly present). Information has also been obtained about the extent to which users are likely to agree on the meaning of a symbol when they first encounter it (see name and concept agreement, below). The database can be used to select symbols on the basis of the relative strengths of particular characteristics required either for experimental or for design purposes. For example, once the precise circumstances under which these dimensions affect symbol usability are known, it may be possible to predict, to a certain extent, the usability of a set of symbols relative to alternative sets on the basis of the relative strength of their characteristics.

ESP ratings are easy to obtain and it is conceivable that users of the database may want to obtain ESP ratings for different sets of symbols and include these symbols and 


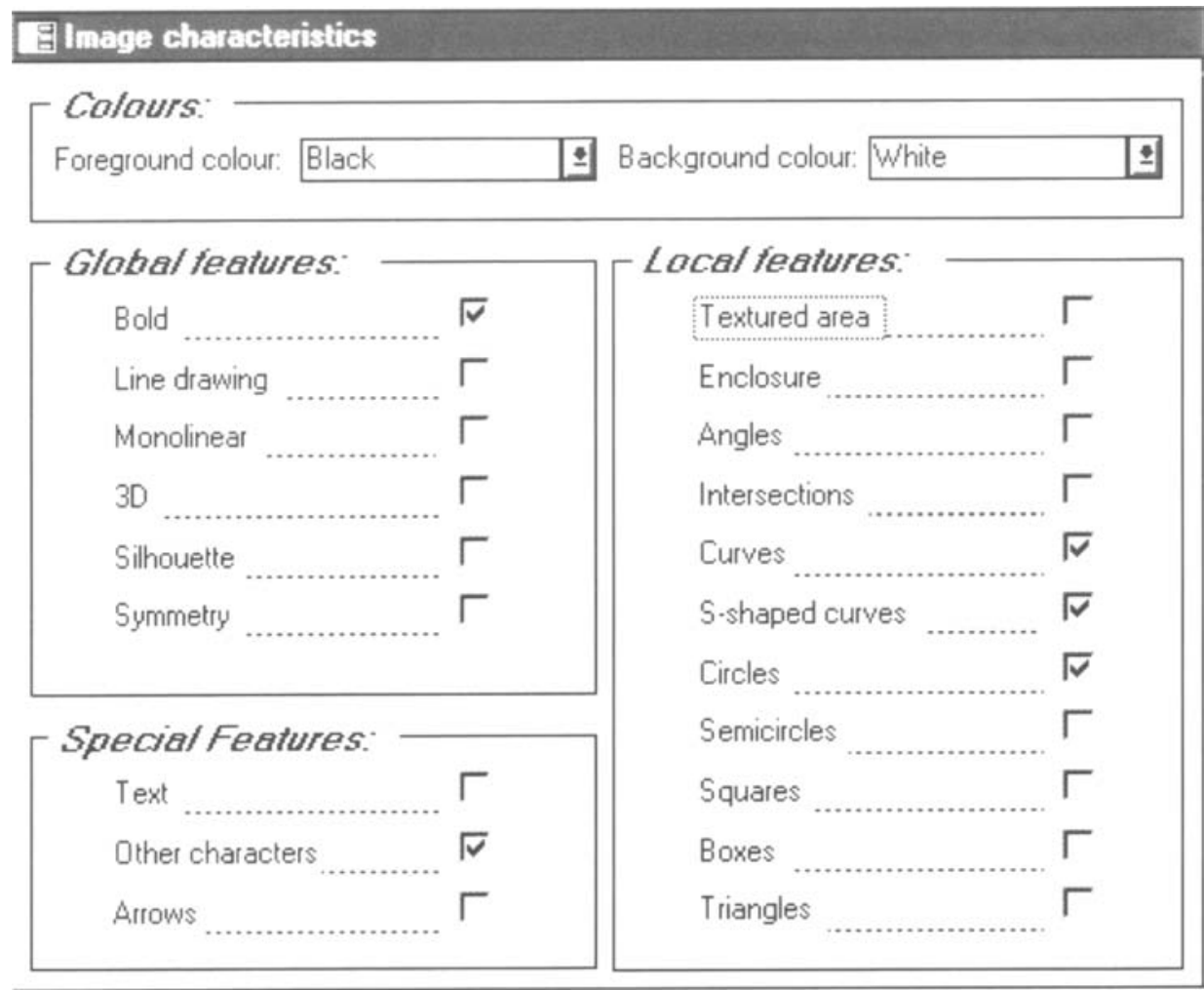

Figure 2. The Image Characteristics Box used to enter and view information about a symbol's graphical features.

their ratings in the database. The way in which each of the ratings was obtained is explained briefly below.

Concreteness. Experimental participants were asked to rate symbols as concrete if they depicted real objects, materials, or people. If these features did not appear in symbols, the symbols were to be regarded as abstract. A 5 -point scale was used to rate concreteness $(1=$ definitely abstract, $5=$ definitely concrete). Participants were encouraged to use all points on the scale including these two extreme values.

Visual complexity. Symbols were regarded as complex if they contained a lot of detail or were intricate, and as simple if they contained few elements or little detail. $(1=$ very simple, $5=$ very complex $)$.

Familiarity. Symbols were rated as "familiar" if they depicted a concept that participants had encountered often before, and as "unfamiliar" if they depicted a concept that participants had never, or rarely, encountered before $(1=$ very unfamiliar, $5=$ very familiar $)$.

Meaningfulness, concept agreement, and name agreement. Ratings of the perceived meaningfulness of symbols were also obtained $(1=$ completely meaningless, 5 = very meaningful indeed $)$.

After rating the meaningfulness of symbols, participants were asked to state briefly what they felt the meaning of the symbol to be. The percentage of participants who were able to ascertain a symbol's designated function, or meaning, was calculated as a measure of each symbol's concept agreement. A further measure, name agreement, was calculated for those symbols for which the most commonly given function differed from the given function. Where concept agreement and name agreement differ, this indicates that there is not a good fit between the symbol and the designated function and that other, better possibilities for a symbol's function exist.

Semantic distance. Symbols vary in their closeness of the relationship between the image and the function being represented. In some cases this relationship is fairly direct (e.g., a printer used as a symbol for printing documents from word processors), whereas in other cases this function is much less direct (e.g., a triangle used to indicate "hazard ahead"). In the first case, the semantic distance between function and symbol might be regarded as quite small, and in the second case, much larger $(1=$ not closely related, $5=$ very strongly related).

AIGA ratings. AIGA ratings were obtained as indications regarding the usability of a set of symbols according to a number of criteria as judged by a panel of professional designers (see AIGA, 1982). These usability criteria are semantic, syntactic, and pragmatic. $S e$ mantic usability refers to the ease with which users can derive a symbol's meaning; syntactic usability refers to the relationship between symbols; and pragmatic usability refers to a symbol's visibility. Thus, AIGA ratings are directly related to forms of usability, whereas the ESP ratings are only indirectly related to usability. The advan- 
tage of ESP ratings is, however, that they can be used in any context in which it is known how ESP ratings relate to usability, whereas AIGA ratings can be used only in the context in which these ratings were obtained.

AIGA ratings are available for only a small number of the symbols presently stored in the database. However, a large number of symbols for which AIGA ratings are available can be obtained from AIGA and put into the database. In addition, these ratings may also be obtained for sets of symbols for which these ratings are not available yet (see AIGA, 1982, for more information about AIGA ratings). The three types of AIGA ratings are described below.

Semantic. The semantic rating refers to the relationship of a symbol to its meaning. When determining this rating, judges were asked to take into consideration questions like (1) How well does this symbol represent its function? (2) Do people from various cultures and ages misunderstand this symbol? (3) Is it difficult to learn this symbol's meaning?

Syntactic. The syntactic rating refers to the relationship between one symbol and another. When determining this rating, judges were asked to take into consideration questions like: (1) How does this symbol look? (2) How well does it relate to other symbols? (3) Is the construction of this symbol consistent with that of other symbols? (4) Are the most important elements recognized first?

Pragmatic. The pragmatic rating refers to the relationship of a symbol to a user. When determining this rating, judges were asked to take into consideration questions like (1) Can a person see the symbol? (2) Is perceptibility of this symbol seriously affected by poor viewing conditions? (3) Is this symbol vulnerable to vandalism? AIGA ratings vary between 1 and 5 , where 1 means that a symbol has few of the characteristics on which the rating is based and 5 means that a symbol has many of the characteristics on which the rating is based.

\section{RETRIEVING SYMBOLS}

Specifying the criteria that symbols have to satisfy in order to be retrieved is done using the Search Form (Figure 3). Each of the symbol characteristics included in the database can be used in the Search Form. The Search Form is divided into six sections, described below. Each search field can be used either in isolation or in combination with other search fields in a single search. However, if more than one search field is used to retrieve symbols, search criteria are combined by the "and" op-

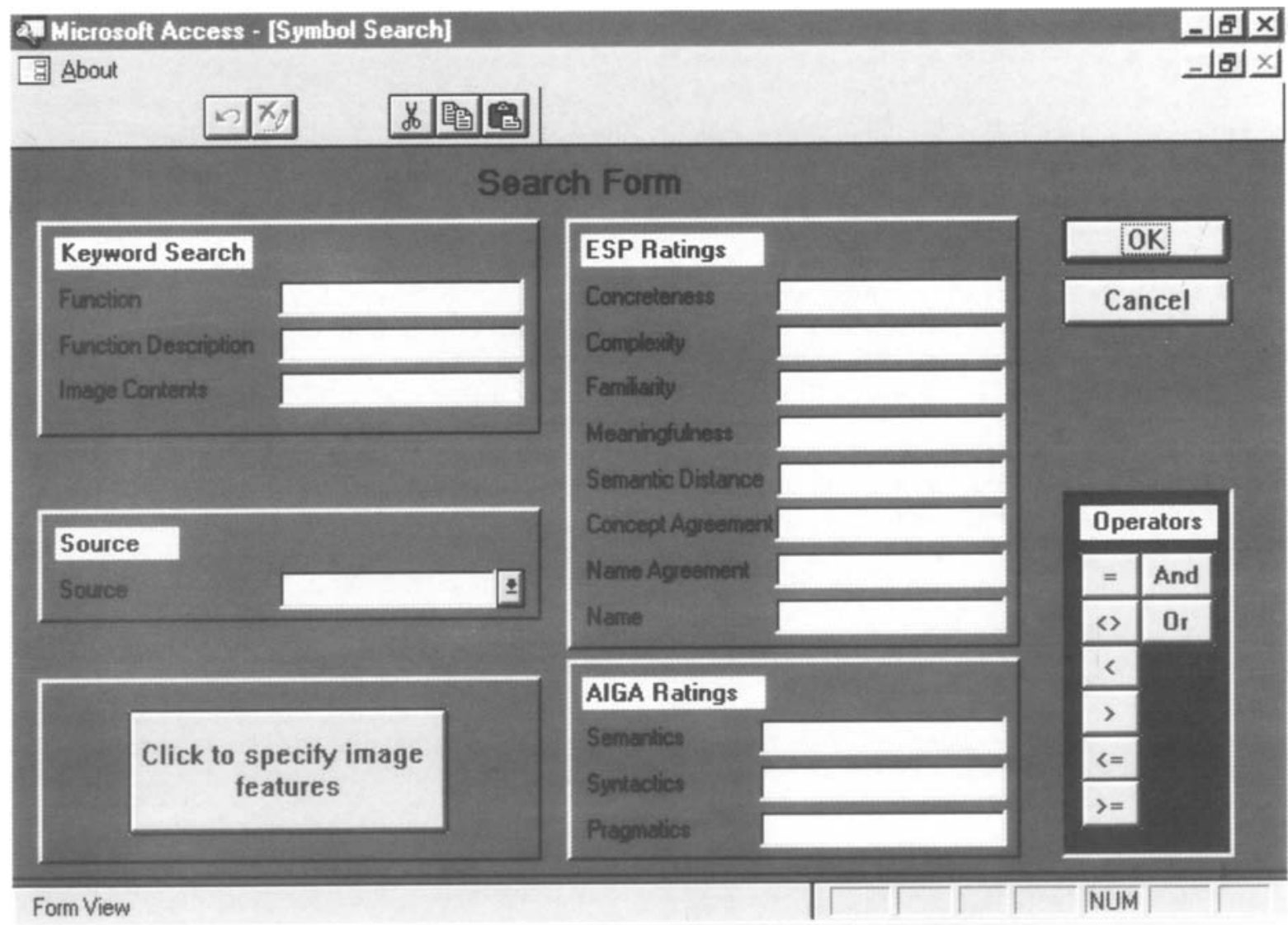

Figure 3. The ESPbase Search Form used to enter search criteria for retrieving symbols from the database. 
erator. For a full explanation of how to use the symbol database in order to construct symbol sets, see the "ESP Symbol Database Users' Manual," available from the authors on request.

\section{Search Form}

Keyword search. Keyword searches can be used to retrieve symbols that contain certain words in their text fields, such as image contents and function fields. For example, typing the word temperature in the function field will retrieve symbols with functions like temperature control and rising temperature.

Source. Using the source field to retrieve symbols allows one to either retrieve all symbols from a single source or to limit other types of searches to retrieve only symbols from the specified source.

Ratings. Symbols can be retrieved on the basis of their ESP and AIGA ratings. This facility has had enormous value to our symbol usability research because it has allowed us to construct, almost at the flick of a switch, symbol sets that vary orthogonally on their concreteness and complexity rating scores (see McDougall et al., 1998). This was achieved simply by specifying ranges for the concreteness and complexity of symbols that fall into the extremes on these scores. A set of symbols that were abstract and simple was obtained by searching for symbols with concreteness and complexity ratings less than 2.5 out of 5 .

Image characteristics. Symbols can be selected on the basis of either the presence or absence of certain image features. This information can be specified in the Feature Specification Box, which pops up if its button in the Search Form is clicked. The Feature Specification Box is similar to the Image Characteristics Box except that a yes/no option is available for each of the local, global, and special features, depending on whether the search is for symbols with these features present or absent. Conceivably, such a search could be used to construct symbol sets in which the symbols are maximally distinctive in terms of shared image features. For example, a set in which all symbols do have curves but do not have angles would be quite distinctive from a set in which all symbols do have angles but do not have curves.

Operators. Arrhythmic operators allow one to specify ranges of rating scores for the symbols to be retrieved. For example, symbols with a familiarity rating greater than 3 can be selected by using the "greater than" operator in the familiarity field. Logical operators allow more complicated searches to be performed by combining search terms within search fields. For example, combining keywords in the function field with the "and" operator allows one to narrow down the number of symbols retrieved relative to using just a single search term.

\section{FINAL COMMENTS}

The ESP symbol database is still under development. Although it has already been an invaluable aid in our re- search, a number of improvements can be made. Some improvements may be prompted by the experimental findings obtained within the Effective Symbology Project at the University of Wales Swansea and other future findings relating symbol characteristics to usability. For example, ratings of symbol distinctiveness (i.e., the amount by which a symbol "stands out" from others) have already been obtained (McDougall et al., 1998), and these ratings could easily be added to the characteristics of symbols already in the database.

In addition to adding more symbol characteristics, it may be possible to incorporate some of the research findings relating symbol characteristics to symbol usability. Inclusion of these findings could potentially lead to the development of a tool for selecting symbol sets that are optimized for usability given the circumstances in which the symbols will be used. For example, if information about the effects of symbol concreteness, complexity, and distinctiveness on symbol usability is incorporated into the database, it may be possible to select symbols that are maximally distinctive and as concrete and complex as the context in which they are to be used dictates.

Notwithstanding possible extensions of the database, however, the abundance of symbol characteristics already included in the database ensures that the ESPbase allows the construction of symbol sets conforming to a host of different criteria. Given the need for research into the effects of a growing number of symbol characteristics on usability, as well as the importance of considering many symbol characteristics in applying symbols in various contexts, the ESPbase may provide useful contributions to both research and design.

\section{Availability}

The database is available from the directly from the authors at the University of Wales Swansea. There is a small cost involved to cover postage and packaging. Running the database requires Microsoft Windows 3.1 or higher and Microsoft Access 2.0 or higher.

\section{REFERENCES}

AmERICAN InStitute of Graphic Arts (1982). Symbol signs: A system of passenger/pedestrian oriented symbols based on an evaluation of existing symbology. New York: Hastings House.

Arend, U., Muthig, K. P., \& Wandmacher, J. (1987). Evidence for global feature superiority in menu selection by icons. Behaviour $\&$ Information Technology, 6, 411-426.

Barnard, P., \& MARCEL, T. (1984). Representation and understanding in the use of symbols and pictograms. In R. Easterby \& H. Zwaga (Eds.), Information design (pp. 37-76). Chichester, U.K.: Wiley.

Benjafield, J., Frommhold, K., Keenan, T., Muckenheim, R., \& Mueller, D. (1993). Imagery, concreteness, goodness, and familiarity ratings for 500 proverbs sampled from the Oxford Dictionary of English Proverbs. Behavior Research Methods, Instruments, \& Computers, 25, 27-40.

BLACKWELL, J. S., \& CuOMo, D. L. (1991). Evaluation of a proposed space and missile warning symbology standard for graphical displays. In Proceedings of the Human Factors Society Thirty-fifth Annual Meeting (pp. 102-106). Santa Monica, CA: Human Factors Society.

Christ, R. E. (1984). Research for evaluating visual display codes: An emphasis on colour coding. In R. Easterby \& H. Zwaga (Eds.), In- 
formation design. The design and evaluation of signs and printed material (pp. 209-228).

ColthearT, M. (1981). The MRC psycholinguistic database. Quarterly Journal of Experimental Psychology, 33A, 497-505.

CRYSTAL VISION (1994). 4000 Icons with Icon Wizard [Computer software]. Carlsbad, CA: CrystalVision Software.

DrEyfuSs, H. (1971). The symbol source book. New York: McGrawHill.

Dyson, M. C. (1992). How do you describe a symbol? The problems involved in retrieving symbols from a database. Information Services \& Use, 12, 65-76.

Familant, E. M., \& Detweiler, M. C. (1993). Iconic reference: Evolv. ing perspective and an organising framework. International Journal of Man-Machine Studies, 39, 705-728.

Friendly, M., Frankl.in, P. E., Hoffman, D., \& Rubin, D. C. (1982). The Toronto Word Pool: Norms for imagery, concreteness, orthographic variables, and grammatical usage for 1,080 words. Behavior Research Methods \& Instrumentation, 14, 375-399.

Garcia, M., Badre, A. N., \& Stasko, J. T. (1994). Development and validation of icons varying in their abstractness. Interacting with Computers, 6, 191-211.

Geiselman, R. E., Landee, B. M., \& Christen, F. G. (1982). Perceptual discriminability as a basis for selecting graphic symbols. Human Factors, 24, 329-337.

Gilhooly, K. J., \& LoGie, R. H. (1980). Age-of-acquisition, imagery, concreteness, familiarity, and ambiguity measures for 1,944 words. Behavior Research Methods \& Instrumentation, 12, 395-427.

GiTTENS, D. (1986). Icon-based human-computer interaction. International Journal of Man-Machine Studies, 24, 519-543.

GRAPHIC ARTISTS GuILd Foundation (1995). Disability access symbols [On-line]. Available HTTP: www.gag.org/das

Gray, L. A. (1990). Icon Manager [Computer software]. Chino, CA: Impact Software.

Green, A. J. K., \& Barnard, P. J. (1990). Iconic interfacing: The role of icon distinctiveness and fixed or variable screen locations. In D. Diaper et al. (Eds.), Human-computer interaction-Interact' 90 (pp. 457-462). Amsterdam: Elsevier.

HORTON, W. (1994). The icon book: Visual symbols for computer systems and documentation. New York: Wiley.

ThE ICON FACTORY (1997). PixelPlace [On-line]. Available HTTP: pixelplace.com

LiU, Z. Q., \& SuN, J. P. (1997). Structured image retrieval. Journal of Visual Languages \& Computing, 8, 333-357.

MARTEIN, R. (1995). Norms for name and concept agreement, familiarity, visual complexity and image agreement on a set of 216 pictures. Psychologica Belgica, 35, 205-225.

MAzza, C. (1997). Neural-net inference and content addressable memory. IEEE Transactions on Neural Networks, 8, 133-140.

McDougall, S. J. P., CuRrY, M. B., \& de Bruij, O. (1998). Understanding what makes icons effective: How subjective ratings can inform design. In M. A. Hanson (Ed.), Contemporary ergonomics 1988 (pp. 285-289). London: Taylor \& Francis.

McDougall, S., Curry, M. B., \& de Bruijn, O. (1999). Measuring symbol and icon characteristics: Norms for concreteness, complexity, meaningfulness, familiarity, and semantic distance for 239 symbols. Behavior Research Methods, Instruments, \& Computers, 31, 487-519.

Mehtre, B. M., Kankanhalli, M. S., \& Lee, W. F. (1997). Shape measures for content-based image retrieval: A comparison. Information Processing \& Management, 33, 319-337.

Paivio, A., Yuille, J. C., \& Madigan, S. A. (1968). Concreteness, imagery and meaningfulness values for 925 words. Journal of Experimental Psychology Monograph Supplement, 76, (3, Part 2).

Quintan, P. T. (1992). The Oxford Psycholinguistic Database [Computer software]. Oxford: Oxford University Press.

RoGERS, Y. (1986). Evaluating the meaningfulness of icon sets to represent command operations. In M. D. Harrison \& A. F. Monk (Eds.), People and computers: Designing for usability (pp. 586-603). Cambridge: Cambridge University Press.

Rogers, Y., \& OBORNE, D. J. (1987). Pictorial communication of abstract verbs in relation to human-computer interaction. British Journal of Psychology, 78, 99-112.

RoHr, G., \& KePPEL, E. (1985). Iconic interfaces: Where to use and how to construct. In H. W. Hendrick \& O. Brown (Eds.), Human factors in organisation design and management (pp. 269-275). Amsterdam: Elsevier.

Sanfeliu, M. C., \& Fernandez, A. (1996). A set of 254 SnodgrassVanderwart pictures standardized for Spanish: Norms for name agreement, image agreement, familiarity, and visual complexity. Behavior Research Methods, Instruments, \& Computers, 28, 537-555.

SNOdgrass, J. G., \& VANDERWART, M. (1980). A standardized set of 260 pictures: Norms for name agreement, image agreement, familiarity and visual complexity. Journal of Experimental Psychology: Human Learning \& Memory, 6, 174-215.

Stammers, R. B., George, D. A., \& Carey, M. S. (1989). An evaluation of abstract and concrete icons for a CAD package. In E. D. Megaw (Ed.), Contemporary ergonomics 1989 (pp. 416-421). London: Taylor \& Francis.

Stammers, R. B., \& Hoffman, J. (1991). Transfer between icon sets and ratings of icon concreteness and appropriateness. In Proceedings of the Human Factors Society Thirty-Fifth Annual Meeting (pp. 354358). Santa Monica, CA: Human Factors Society.

van Schagen, I., Tamsma, N., Bruggemann, F., Jackson, J. L., \& MICHON, J. A. (1983). Namen en normen voor plaatjes [Names and norms for pictures]. Nederlands Tijdschrift voor de Psychologie, 38, 236-241.

WILSON, M. (1988). MRC Psycholinguistic Database: Machine-usable dictionary, version 2.00. Behavior Research Methods, Instruments, \& Computers, 20, 6-10.

\section{NOTES}

1. Unless otherwise specified, the term symbol refers to icons, pictograms, symbols, and signs.

2. Microsoft Access is protected by copyright held by the Microsoft Corporation. Microsoft Windows is a registered trademark of the Microsoft Corporation.

3. In order to save disk space, the original image files were RLE encoded before being embedded into the symbol database. Encoding reduces their file down to approximately $10 \%$ of the non-encoded image files. The number of different colors used in the symbol images was limited to 16 for the same reason.

(Manuscript received December 5, 1997; revision accepted for publication April 23, 1998.) 